\title{
UNA APROXIMACIÓN A “ÁNGEL DE OCONGATE” DE EDGARDO RIVERA MARTÍNEZ
}

\section{Jorge Valenzuela Garcés}

Universidad Nacional Mayor de San Marcos

jorgevalenzuela4@hotmail.com

RESUMEN: El objetivo de este artículo es observar el modo en que el tratamiento de la identidad, en "Ángel de Ocongate" de Edgardo Rivera Martínez, se realiza a partir de la elección del ángel, ser mítico perteneciente a la tradición cristiana occidental. Realizamos el análisis a partir de un acercamiento a la naturaleza del personaje principal y a su subjetividad.

PALABRAS CLAVE: “Ángel de Ocongate”, Edgardo Rivera Martínez, cuento peruano, literatura fantástica.

ABSTRACT: The purpose of this article is to observe how Peruvian writer Edgardo Rivera Martínez treats the theme of identity in his story "Angel de Ocongate" through the creation of an angel, mythological being pertaining to the Western Christian tradition. This analysis is based on an approach that reveals the protagonist's personality and his subjective perspective on life.

KEYWORDS: “Angel of Ocongate”, Edgardo Rivera Martínez, Peruvian short story, fantastic literature

\section{Introducción}

Una de las características de la narrativa inicial de Edgardo Rivera Martínez (pensemos en textos como "El visitante" o "Ángel de Ocongate") es haber tratado el tema de la identidad a través de las estrategias propias de la narración fantástica que incorpora elementos de origen mítico como el personaje angélico. En efecto, en estos cuentos podemos observar el modo en que Rivera Martínez atenta contra cualquier discurso monolítico sobre la noción de identidad para revelarnos, a través del proceder fantástico, las contradicciones de un sujeto (en los dos cuentos referidos un ángel) que se encuentra en permanente meditación, interrogación, soledad y destierro en contextos que les son ajenos (un malecón neblinoso y la puna andina respectivamente) después de haber perdido su condición sagrada para no volver a recuperarla más.

A partir de un personaje como el ángel, la narrativa inicial de Rivera Martínez privilegia la representación de los marginales sociales incidiendo en aspectos que los hace únicos, incomparables y singulares, buscando, de ese modo, acercarse metafóricamente a nuestra condición de seres humanos marcados por la defectividad.

En sus cuentos, estos seres son individualidades que relativizan el discurso fuerte de los que proclaman la existencia de una "identidad" homogeneizadora (Morillas 311 y sgtes). En este sentido, su narrativa se construye sobre esta clase de personajes cuya identidad en 
proceso de disolución, está en consonancia con espacios (neblinosos y oscuros como en "El visitante") en los que lo difuso también es la regla y en los que el trasfondo étnico e histórico, sobre todo si pensamos en un cuento como "Ángel de Ocongate", cumple con hacer más crítica y confusa la construcción de un sujeto "dueño" o poseedor de una identidad.

\section{EI personaje angélico en Edgardo Rivera Martínez}

Una de las características de un texto como "Ángel de Ocongate", objeto de nuestro análisis, es haber abordado el problemático horizonte de la subjetitivad y sus formas de construcción a través de una narración que incorpora un personaje híbrido (en este caso un ángel-danzante) cuya función es poner en evidencia una crisis identitaria a partir de las contradicciones generadas a partir de la doble condición sagrada y profana que lo caracteriza.

En efecto, en el cuento tenemos, de un lado, a personaje cuya primera faz se articula a un origen mítico, configurado a partir de un ángel inmortalizado en el friso de una capilla abandonada y, de otro, a un danzante cuyo rostro nos muestra a un humano, un danzante cuyo desconcierto lo ha llevado por parajes desolados del ande preguntándose sobre sí mismo después de haber perdido su condición angélica.

A partir de este cuento, emblemático dentro de la producción de Rivera Martínez, es posible observar el modo en el escritor jaujino ha centrado su atención en el cuestionamiento de cualquier discurso monolítico sobre la noción de identidad y el papel activo que cumple la construcción de la subjetividad (Vich: 2008) en el contradictorio proceso que nos lleva a definir a un sujeto que sufre la pérdida del sí mismo y experimenta un vaciamiento de su primera y prístina condición.

En "Ángel de Ocongate" la figura del ángel le sirve a Rivera Martínez para la representación del marginal, incidiendo en aspectos que lo hacen único e incomparable. En efecto, además de su condición única, nos encontramos con un personaje en permanente soledad, destierro, meditación, e interrogación sobre su origen, condición y destino.

Sirva, pues, este breve cuento, para advertir las constantes de la obra de Rivera Martínez construida sobre un repertorio de personajes cuya indeterminación identitaria está en consonancia con la proyección de un universo interior conflictivo (en el que resonancias culturales occidentales como andinas están en permanente confrontación) y con la construcción de escenarios (en los que cunde el abandonado o la esterilidad) que cumplen con hacer más crítica y confusa la construcción de un sujeto "dueño" de sí mismo y de su entorno. Por estas razones, en la narrativa inicial de Rivera Martínez, será central el tratamiento de personajes cuya relación con la realidad se torne confusa y contradictoria, marcada por el deseo, siempre insatisfecho, de aprehenderla.

Harold Bloom sostiene en su bello libro El ángel caído que los ángeles "solo tienen sentido si representan algo que era nuestro y que podemos volver a ser" (2008: 33). Es decir, Bloom nos habla de un proceso de recuperación de lo perdido, proceso que sin duda entraña una búsqueda cuyo éxito no puede ser asegurado, pero que depende de lo que los ángeles pueden llegar a representar en su contacto con lo humano. Lo que Bloom llama lo nuestro y su recuperación estaría implícitamente relacionado con lo que, al haber sido perdido por el hombre, todavía puede ser encarnado o advertido en un ángel, algo que al perderse supuso un empobrecimiento de nuestra condición, pero que está allí, latente, presto a ser recuperado. Esta posibilidad de volver a ser lo que fuimos advertida por Bloom 
debería ser ligada a la esperanza, ese posible siempre abierto que sostiene nuestra existencia y que los ángeles, como ningún otro ser, pueden materializar.

Ampliando la idea de Bloom podría postularse que los ángeles tienen sentido para el hombre si representan algo que potencialmente podemos ser, es decir, algo que ellos pueden representar en su infinita capacidad de ser y en el ejercicio de sus grandes poderes y que está en nosotros desarrollar. Los ángeles, así, serían el instrumento no solo de la recuperación de lo perdido sino un medio para ampliar nuestra condición expuesta a una permanente transformación y enriquecimiento.

Estas ideas nos permiten comprender por qué en la narrativa de Rivera Martínez los ángeles que pueblan sus historias se encuentren en permanente búsqueda e intento de recuperación de lo perdido a pesar del desconcierto en el que viven sumidos. Algunos de ellos mantienen su condición seráfica, pero afectados por el contacto con humanos como en "El visitante", otros como en "Ángel de Ocongate" sufren la pérdida de su propia condición de ángeles haciendo aún más arduo y hasta imposible el camino de regreso a su condición primigenia.

En el marco de la cuentística fantástica maravillosa de Rivera Martínez (en el que nada queda explicado y la experiencia de lo real deviene perturbadora y desestabilizante), la presencia de un ángel (entidad de naturaleza mítica) problematiza el concepto de identidad al plantear radicalmente un escenario en el que, como dijimos, el ángel protagonista ha dejado de serlo para asumir, en su nueva condición humana, la apariencia de un danzante andino. Así, en este cuento, el programa narrativo del personaje protagonista se plantea a partir de un estado inicial de conjunción con el objeto de valor, en este caso, la identidad angélica, para sufrir, luego, su pérdida, a partir de una acción violenta de la que no es responsable (la sufre pasivamente y no la recuerda), y que en el relato adopta la forma de un rayo que propicia su caída del friso en el que se encontraba en perfecta armonía con otros tres ángeles danzantes. La pérdida de la condición angélica busca hacer evidente, en el ángel danzante ya humanizado, la nueva condición de un ser que, ahora, sufre los rigores del dolor y la nostalgia.

De otro lado, es posible explicar la elección del ángel como personaje protagónico en la narrativa inicial de Edgardo Rivera Martínez debido al carácter singular de la existencia de este ser mítico y la riqueza que comporta al poseer una identidad problemática generada por sus permanentes desplazamientos del orden celestial al terreno, identidad que, además, por su carácter móvil e híbrido permite la indagación en un horizonte cultural como el peruano caracterizado por constantes intersecciones entre la tradición cristiana y la andina.

En el caso de "Ángel de Ocongate" estamos frente a un ángel ya humanizado, desprovisto de esa santidad cristiana que la tradición religiosa les ha atribuido como marca de nacimiento. En el cuento este ángel ha perdido su función esencial de asistir o servir a Dios o portar mensajes celestiales orientados a introducir la civilización cristiana en el mundo. Este ángel ya no es puro, en un sentido estricto, ni posee una gran capacidad que le permita ejercer un eficiente desempeño entre los hombres. De hecho, en el cuento concentra en sí mismo solo angustia y confusión.

En efecto, en el cuento sucede que este ángel caído ya humanizado es un ser cuyo contacto con los humanos problematiza su situación personal llevándolo a los márgenes de la incertidumbre y la desesperanza pero, fundamentalmente, a experimentar el sentimiento de soledad. Es, pues, un ser individualizado y solitario que, en el contacto con los seres humanos, empieza a conocerse a sí mismo, lo que implicaría la recuperación de una subjetividad, esta vez humana, interpersonal, (de allí su gran capacidad para razonar), pero 
cuya condición no cambiará ni se resolverá en un estado de yunción con el objeto de valor perdido, esto es, su condición angélica.

En el cuento "Ángel de Ocongate" sucede que el ángel protagonista no cumple con la labor de proteger a los seres humanos, ni de convertirse en guardián de alguien, ni de ser portador de la luz divina. En este caso estamos, incluso, ante un ángel que se observa a sí mismo como una "apagada sombra", como "ave negra" cuya ignorancia, con respecto a la razón de su "caída" será eterna, así como su existencia errante.

A nivel práctico, este ángel no es dador de vida, ni anuncia la llegada del hijo de Dios como en los cuadros del Renacimiento. Apenas si puede comprender el lugar que le correspondió ocupar en un mundo anterior, antes de perder su condición sagrada. Por ello solo existe como una pura subjetividad que se hace manifiesta en el acto de enunciación desde el discurso de la duda y la interrogación constante.

Cabría anotar, para complementar estas apreciaciones sobre "Ángel de Ocongate", que los ángeles en la narrativa inicial de Rivera Martínez carecen de la capacidad del autorreconocimiento. Están despojados de la capacidad de la autocontemplación. Su imagen no se refleja en los espejos como en el caso de "El visitante" o básicamente no saben quiénes son como en "Ángel de Ocongate". De hecho, aquello que los cristianos denominan "alma" es algo de lo que carecen y reemplazan con el logos, con la razón. De otro lado, les es ajena la cualidad de la perfección, aunque las descripciones sobre ellos, como en un relato como "Ángel de Ocongate", los describan como seres poseedores de una gran belleza y juventud.

Es importante anotar, asimismo, que la presencia de los ángeles en los cuentos de Rivera Martínez se sostiene sobre la base de la fascinante presencia del ángel en la cultura occidental y en la pintura y arquitectura coloniales del Barroco peruano. Esta conexión con el Barroco es imprescindible para entender, en un primer nivel, por qué estos seres imbuidos de una metafísica personal son también una mezcla, un producto híbrido que encarna una permanente búsqueda del sí mismo en un contexto de crisis. En otro nivel, nos permite comprender el horizonte mestizo que, en un cuento como "Ángel de Ocongate", posibilita la configuración de un ángel que es a la vez un danzante o dansak.

\section{EI proceso de desacralización en "Ángel de Ocongate"}

Es posible postular que en la base del desajuste identitario del personaje central (Vich, 2008), por lo menos a partir de los elementos que presenta el cuento, se encuentra, en un primer nivel, un proceso de desacralización, de desmitificación cambio que supone la pérdida de la condición angélica. En "Ángel de Ocongate” tenemos a un ángel (visto como un héroe civilizador según Eliade (1967:95), que ha dejado de serlo para convertirse en un humano con el aspecto de un danzante andino.

Este hecho, digamos el paso de una existencia mítica a una humana, da pie al nacimiento de la subjetividad del personaje que en el cuento es presentada o explicada, por quienes lo observan, a partir de la pérdida de la memoria. De esta forma, el mito del ángel caído convertido en humano se actualiza en el horizonte andino y busca sentar las bases de un sujeto en cuyo origen se pueda advertir, de un lado, la pérdida de una condición, digamos angélica o sagrada, y a la vez el nacimiento de una subjetividad cuyo rasgo central se dará a partir de la imposibilidad del autorreconocimiento y la pérdida de sentido. Citemos: "Y como yo callaba y notaban el raro fulgor de mis pupilas, y mi abstraimiento, mi melancolía, acabaron por considerar que había perdido el juicio a la vez que la memoria...". Es decir, 
en el cuento se construye un escenario que presenta a un sujeto inestable configurado por un no saber sobre sí mismo a partir de la pérdida del orden mítico (los ángeles representados en el friso de la capilla) orden dentro de cual, por ejemplo, la actividad de la danza de la que participaba como ángel tenía sentido, ese sentido que, en el presente del relato, se ha perdido. Citemos: "Qué baile es el que danzan? ¿Qué música la que siguen? ¿Es el suyo un acto de celebración y de alegría?"

Es pertinente anotar que el cuento busca destacar el nacimiento de la subjetividad del personaje como un acontecimiento ocasionado a partir de una pérdida (hecho central que determina que la subjetividad del danzante esté marcada por la imperiosa necesidad de recuperar el sentido) configurada en el cuento a partir de un permanente deambular en el que se ignora quién se es y el futuro que se avizora como un destierro permanente.

Un aspecto que refuerza el proceso de desacralización en el texto es el relacionado con el escenario principal en el que se desarrolla los hechos. Nos referimos a la capilla en ruinas y al atrio en el que se encuentra el ángel monologante. De acuerdo con Eliade una basílica, una catedral o un templo, constituyen "la reproducción terrestre de un modelo trascendente" (1967:63) en este caso, el modelo de un espacio sagrado celestial. Si, según Eliade, la pertinencia de un tempo como casa de Dios o de los dioses supone una resantificación continua del mundo (1967:63) o la sacralización de un espacio libre de toda corrupción, en el cuento de Rivera Martínez observamos una pérdida absoluta de esa función sagrada. Citemos un pasaje del cuento: "Quién soy sino apagada sombra en el atrio de una capilla en ruinas, en medio de una puna inmensa".

Abandonada a su suerte la capilla de la Santa Cruz ha perdido su función resantificadora y congregadora de la grey. Lejos de purificar a quienes asistan a ella o de trasmitir a los creyentes el orden de un mundo celestial, la capilla abandonada proyecta la imagen de la destrucción y del caos que se prolonga a la conciencia del ese ángel-dansak perdido. Citemos: "Y así, después de tres jornadas, llegué a ese santuario abandonado, del que apenas quedan la fachada y los pilares". Si a esta situación defectiva sumamos el hecho de que este espacio sagrado ha sido profanado por un rayo (despojando de su condición angélica a uno de sus danzantes), tenemos los elementos suficientes para explicarnos el sinsentido en el que se encuentra sumido el mundo representado y, por ende, el protagonista. De este modo que creemos que el cuento logra plasmar la disolución del sujeto en consonancia con un entorno que se presenta a sí mismo en un proceso de descomposición.

\section{Identidades negadas, subjetividades amenazadas en "Ángel de Ocongate"}

Las definiciones más simples de sujeto, las que encontramos en el Diccionario de la Real Academia Española, nos indican que sujeto es quien se encuentra expuesto o propenso a algo. Otra acepción, de índole peyorativa, refiere la utilización del término sujeto cuando se ignora el nombre de alguien o cuando deliberadamente no se quiere declarar de quien se habla. La primera acepción, la que nos interesa, refiere una situación de vulnerabilidad relacionada con la exposición a cualquier situación que precipite la pérdida de los referentes a partir de los cuales nos instalamos en el mundo.

De otro lado, las actuales y más difundidas definiciones de sujeto establecen que este se constituye sobre la base de relaciones intersubjetivas y que, por lo tanto, el vínculo del sujeto (expuesto) en relación con el otro, produce en él distintos tipos de subjetividades. Es 
a partir de estas subjetividades que el sujeto empieza su dura tarea de ordenarse psíquicamente en un mundo complejo. De este modo, el sujeto construye su propia identidad en sus vínculos con el otro, pero también cuando se responde a las demandas de sus deseos o a sus propias necesidades de autorreconocimiento.

El ángel-dansak que protagoniza la historia de "Ángel de Ocongate" es un ser que, al haber caído, ha perdido su condición angélica afectando, de ese modo, aquello que la sostenía y articulaba. En efecto, el ángel protagonista del cuento de Rivera Martínez, al dar paso violentamente al nacimiento de su subjetividad después de su caída (nos referimos a una subjetividad que empieza a ser problemática) se ha visto en la necesidad de reconstruirse a sí mismo apelando a la memoria colectiva, representada, en el cuento, por el hombre viejo que le habla en quechua y por el discurso que, sobre él, construyen los otros, un discurso constituido, básicamente, por preguntas. Es, sin embargo, el anciano quien lo reidentifica y le otorga un pasado, esa memoria que, se supone, ha perdido. Citemos del cuento: "No había nadie sino un hombre viejo que descansaba y que me miró con atención. Me habló de pronto y me dijo en un quechua que me pareció muy antiguo: "Eres el bailante sin memoria. Eres él y hace mucho tiempo que caminas. Anda a la capilla de la Santa Cruz, en la pampa de Ocongate. ¡Anda y mira!”

En este acto de comunicación se funda todo el horizonte identitario del ángel-danzante. En este sentido, antes de recibir la información del anciano, el ángel-danzante es un ser vaciado de sí mismo, despojado del horizonte mítico en el que tenía pertinencia su existencia. En este momento cabe hacerse la siguiente pregunta: $¡$ a qué tipo de subjetividad nos referimos en el caso del ángel protagonista del cuento de Rivera Martínez si, según Foucault, solo podemos hablar de subjetividades a partir del ejercicio de prácticas sociales y epistémicas?

Desde este punto de vista cabe postular que el cuento presenta de manera paradigmática la negación de cualquier principio esencialista en torno a la idea de sujeto cuya trayectoria, como se aprecia en el cuento, simplemente se inicia con un acto de comunicación que resulta fundante. De ese modo el cuento da paso a la escenificación de un momento primordial en el que la subjetividad del ahora danzante nace a partir del contacto con el otro. El discurso del anciano que reconoce al ángel caído, sirve al propósito de construir una subjetividad, la del ángel danzante (cuyo antecedente mítico refiere un estado de bienestar irrecuperable) marcada a fuego por el sentimiento de pérdida. Es así como el cuento construye su discurso, un discurso que se formula sobre la base de las primeras impresiones, intuiciones de los otros sobre él y el saber del anciano que le dirige la palabra para informarle sobre sí mismo y su procedencia.

Manipulado, entonces, por el anciano en el propósito de reconocerse así mismo y saberse poseedor de una identidad, al ángel danzante solo le queda confirmar quién es y de esa manera poseer, finalmente, su objeto de valor.

De acuerdo con el texto, el protagonista de "Ángel de Ocongate" es reconocido por un anciano de Raurac como "el bailante sin memoria". Estamos pues, ante un danzante cuya apariencia es la de un ángel despojado de su pasado y de su futuro, instalado en un presente que lo inmoviliza y solo produce en él un conjunto de preguntas después de haber sufrido una caída del friso de la capilla en el que se mantenía como una figura mítica. Eso es lo que, gracias al texto, junto con el protagonista, descubrimos hacia el final del relato como una gran revelación. A través del cuento podemos observar el friso impactado por el rayo que ha causado su caída. Citemos: "Y allí, en la losa quebrada otrora por un rayo, hay cuatro figuras en relieve. Cuatro figuras danzantes". 
La construcción del personaje central como ángel-danzante nos permite advertir los problemas que porta una identidad problemática que, en este caso, se despliega, en un primer nivel, para acercar al ángel de la tradición mítica occidental al universo andino, revestido, como está, de un ropaje propio de los ángeles de la tradición de la pintura de la escuela cuzqueña y, en un segundo nivel, para hacer complejos los procesos de autorreconocimiento del propio protagonista, más aún, si como sucede en el relato, ha perdido la memoria después de la caída del friso en el que danzaba.

Las referencias en el cuento a la imposibilidad del ángel de reconocer la música de las quenas y los sicuris y las melodías de las cuadrillas de músicos con los que se encuentra en los pueblos andinos en fiesta, inciden en un aspecto central de su configuración como actor, esto es, la incapacidad de reconocerse culturalmente en el otro y por lo tanto de autorreconocerse. Citemos del cuento: "Me conmovían sus interpretaciones, más no reconocía jamás una melodía ni hallé una vestimenta que se asemejara a la mía".

Este proceso de clausura, este cierre de la comunicación sume a este ángel monologante en un estado cuyo carácter mórbido se manifiesta en esa constante interrogación que caracteriza sus evoluciones mentales, en el aspecto descuidado de su presencia y en su vagar sin fin por "los caminos y los páramos, sin dormir y sin acordarme de un principio ni avizorar una meta". Este estado puede ser descrito como una pérdida del espíritu, como un vaciamiento de la memoria que simbólicamente se figura en el cuento como una caída en los abismos de la despersonalización. Incidamos en el hecho de que el efecto de la caída animiza y corporiza al ángel cuyo anterior estado podemos asociar al de una figura de piedra.

\section{Breve aproximación a las relaciones entre identidad y fantástico en "Ángel de Ocongate"}

Es importante destacar, como lo hace Enriqueta Morillas en su artículo "Identidad y literatura fantástica" (ver bibliografía) que, al ser tratado el tema de la identidad en el ámbito de lo fantástico, la mayoría de los personajes "adolecen del singular phatos de la irrealidad y se sitúan, al parecer de manera inexorable, frente a una situación que se presenta como una suerte de Jano bifronte, cuya andadura deberá transitar los senderos que propone la doble articulación del texto" (1999: 318). En esta dirección el empleo de la disociación, esto es, de la suspensión de la habitual unidad y correspondencia entre el sujeto y el sí mismo es clave para entender el proceder fantástico cuando se aborda el tema identitario (Morillas: 1999:318)

De otro lado, Morillas se refiere a la "transgresión de la frontera entre lo animado y lo inerte, de la vigilia y del sueño" (1999: 319) para reforzar la disociación del sujeto inserto en la situación problemática que el fantástico siempre escenariza, situación que instalará la duda tanto en el lector implícito como en el protagonista del relato. En efecto, el paso a lo animado o a la humanización de entidades míticas o de objetos inertes y la dinámica del despertar y el dormir como un mecanismo que vincula dos instancias tempo espaciales, son mecanismos útiles al propósito de desestabilizar o cuestionar los límites impuestos por las leyes naturales dentro de las cuales nos movilizamos (Morillas:319) A esto debemos sumar el empleo de diversas figuraciones como el doble, los gemelos y los espejos y algunos procedimientos como la repetición y el desdoblamiento que permiten, por ejemplo, la convivencia de dos instancias temporales con dos sujetos que al final son el mismo, como 
en "El otro" de Borges, o la capacidad para multiplicar al infinito la imagen de la persona vulnerando de esa manera su integridad.

Como es evidente hasta aquí, queda claro que la imagen del mundo construida a través del proceder fantástico, vinculado con el tema de la identidad, se ve afectada, básicamente, en sus dos dimensiones. La primera, la espacial, que se relativiza o se vuelve desconocida para el personaje y, la segunda, la temporal, que supone un discurrir inédito, conflictivo mediante el cual el protagonista del relato puede llegar a sufrir una severa confusión con respecto al momento en que se encuentra. De hecho, en el cuento analizado, la instancia temporal de la enunciación es el presente despojado de pasado y de futuro, como mencionábamos líneas arriba. Luego, es lógico que, el lugar desde el cual se genera el sentido, esto es, la mente del protagonista, se vea afectada. De este modo, que ya sea a través de la incertidumbre, la confusión o la pérdida de la memoria, el personaje no reconoce un lugar en el mundo que le sea familiar ni propicio. Es el momento en que se produce una clausura de la subjetividad y, por ende, de la identidad del sujeto.

En “Ángel de Ocongate”, el ángel-danzante trata, en primer término, de intuir su identidad con los pocos elementos que le han sido otorgados. El propósito del autor es situarnos, desde la duda compartida por el protagonista, en una problemática de hondas resonancias étnicas, sociales y culturales no resueltas en él y por ende en la sociedad a la que pertenece como un no integrado al centro desde donde irradia el poder. Instalado en su propia soledad y en medio de un paraje inhóspito, este ángel danzante continúa en el camino de su exilio ignorando la razón de su desgracia y por ello sin saber quién es en realidad. Sufre lo que Morillas define como síndrome de irrealidad (1999: 308), sobre todo porque ha pasado de un nivel de realidad a otro, pero sobre todo porque en el nivel en que se encuentra todo ha perdido sentido.

Al actualizar el mito occidental del ángel caído y al acercarlo a nuestra tradición, revestido con los ornamentos del danzante andino, Rivera Martínez combina dos planos: el mítico y el realista. Así, en este cuento es posible observar, emblemáticamente, la manera en que lo fantástico entra a dialogar con nuestro pasado colonial y con las construcciones sobre lo mestizo. En efecto, "Ángel de Ocongate” refunda una mitología, la cristiana, al transformar al ángel bíblico en un ángel danzante de los andes cuya identidad resulta un enigma para el propio protagonista, esto es, una pregunta sin respuesta: "Sí, sombra soy, apagada sombra. Y ave, ave negra, que no sabrá nunca la razón de su caída. En silencio siempre, y sin término la soledad, el crepúsculo, el exilio".

\section{REFERENCIAS BIBLIOGRÁFICAS}

BLOOM, Harold (2008): El ángel caído. Barcelona, Paidós.

ELIADE, Mircea (1967): Lo sagrado y lo profano. Madrid: Ediciones Guadarrama S.A.

FERREIRA, César (2006): Edgardo Rivera Martínez: Nuevas Lecturas. Lima, Fondo Editorial de la Universidad Nacional Mayor de San Marcos.

MORILLAS VENTURA, Enriqueta (1999): "Identidad y literatura fantástica" en Anales de literatura hispanoamericana, 28; pp. 311-321.

REISZ, Susana (1982): "Borges: Teoría y práctica de la ficción fantástica” en Lexis, vol VI, 2, p.p. 161-202. 
RIVERA MARTÍNEZ, Edgardo (2006): "Lo fantástico y lo maravilloso en mis primeros relatos" en César Ferreira (editor) Edgardo Rivera Martínez: Nuevas lecturas. Lima: Fondo Editorial de la Universidad Nacional Mayor de San Marcos, pp. 69-72.

RIVERA MARTÍNEZ, Edgardo (2004): Cuentos completos. Lima, INC.

RIVERA MARTÍNEZ, Edgardo (1986): Ángel de Ocongate y otros cuentos. Lima, Peisa.

VICH, Cynthia y Víctor VICH (2001): "La taciturna corriente que me absorbe": "Ángel de Ocongate y el problema de la identidad en el Perú" en Revista de crítica literaria latinoamericana 54, pp. 163-169.

VICH, Cynthia (2008): "Ángel de Ocongate" de Edgardo Rivera Martínez: apuntes para una exploración sobre la construcción del sujeto". Consultado en http://www.fas.harvard.edu/ icop/cynthiavich.html, el 20 de marzo del 2015.

(C) Jorge Valenzuela Garcés

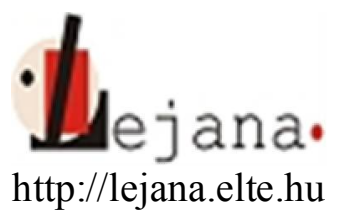

Universidad Eötvös Loránd, Departamento de Español, 1088 Budapest, Múzeum krt. 4/C

Recibido: 04 de agosto de 2015

Aceptado: 14 de octubre de 2015 